\title{
Cis-Acting Sequence
}

National Cancer Institute

\section{Source}

National Cancer Institute. Cis-Acting Sequence. NCI Thesaurus. Code C13370.

Cis-acting locus is a section of DNA that affects the activity of DNA sequences on that same molecule of DNA. 